[7] B. R. Barmish and H. I. Kang, "A survey of extreme point results for robustness of control systems," Automatica, vol. 29, pp. 13-35, 1993.

[8] L. Jetto, "Strong stabilization over polytopes," IEEE Trans. Automat. Control, vol. 44, no. 6, pp. 1211-1216, Jun. 1999.

[9] Ü. Nurges, "New stability conditions via reflection coefficients of polynomials," IEEE Trans. Automat. Control, vol. 50, no. 9, pp. 1354-1360, Sep. 2005.

[10] Ü. Nurges, "Robust pole assignment via reflection coefficients of polynomials," Automatica, vol. 42, pp. 1223-1230, 2006.

[11] D. Henrion, M. Sebek, and V. Kucera, "Positive polynomials and robust stabilization with fixed-order controllers," IEEE Trans. Automat. Control, vol. 48, no. 7, pp. 1178-1186, Jul. 2003.

[12] H. Khatibi, A. Karimi, and R. Longchamp, "Fixed-order controller design for polytopic systems using LMIs," IEEE Trans. Automat. Control, vol. 53, no. 1, pp. 428-434, Feb. 2008.

[13] J. L. Diaz-Barrero and J. J. Egozcue, "Characterization of polynomials using reflection coefficients," Appl. Math. E-Notes, vol. 4, pp. 114-121, 2004.

[14] S. M. Kay, Modern Spectral Estimation . Englewood Cliffs, NJ: Prentice Hall, 1988.

[15] A. M. Oppenheim and R. W. Schaffer, Discrete-Time Signal Processing. Englewood Cliffs, NJ: Prentice-Hall, 1989.

[16] F. Kraus, B. D. O. Anderson, and M. Mansour, "Robust Schur polynomial stability and Kharitonov's theorem," Int. J. Control, vol. 47, pp. 1213-1225, 1988

[17] B. Picinbono and M. Benidir, "Some properties of lattice autoregressive filters," IEEE Trans. Acoust. Speech Signal Process., vol. 34, no. 2, pp. 342-349, Apr. 1986.

[18] B. Büeler, A. Enge, and K. Fukuda, "Exact volume computation for polytopes: A practical study," Polytopes-Combinatorics Computat., pp. $1-18,1998$.

[19] A. C. Bartlett, C. V. Hollot, and L. Huang, "Root location of an entire polytope of polynomials: It suffices to check the edges," Math. Control, Signals Syst., vol. 1, pp. 61-71, 1988.

\section{Homography-Based Visual Servo Control With Imperfect Camera Calibration}

\author{
G. Hu, W. MacKunis, N. Gans, W. E. Dixon, J. Chen,
} A. Behal, and D. Dawson

Abstract - In this technical note, a robust adaptive uncalibrated visual servo controller is proposed to asymptotically regulate a robot end-effector to a desired pose. A homography-based visual servo control approach is used to address the six degrees-of-freedom regulation problem. A highgain robust controller is developed to asymptotically stabilize the rotation error, and an adaptive controller is developed to stabilize the translation error while compensating for the unknown depth information and intrinsic camera calibration parameters. A Lyapunov-based analysis is used to examine the stability of the developed controller.

Index Terms-Lyapunov methods, quaternion representation, robust adaptive control, uncertain systems, visual servo control.

\section{INTRODUCTION}

Image-based feedback continues to be a popular sensor modality for autonomous control applications. A camera model (e.g., the pinhole model) is often required in these applications to relate image-based feedback to the (normalized) Euclidean-space. The camera model is typically assumed to be exactly known (i.e., the intrinsic calibration parameters are assumed to be known). Despite the availability of several popular calibration methods, camera calibration can be time consuming, requires some level of expertise, and has inherent inaccuracies. If the calibration parameters are not exactly known, then performance degradation and potential unpredictable response from the system may occur.

Motivated by the desire to incorporate robustness to camera calibration, different control approaches that do not depend on exact camera calibration have been proposed (cf. [1]-[18]). Efforts such as [1]-[5] have investigated the development of methods to estimate the image and robot manipulator Jacobians. These methods are composed of some form of recursive Jacobian estimation law and a control law. Specifically, a visual servo controller is developed in [1] based on a weighted recursive least-squares update law to estimate the image Jacobian. In [2], a Broyden Jacobian estimator is applied and a nonlinear least-square optimization method is used for the visual servo control development. In [3], the authors used a nullspace-biased

Manuscript received June 26, 2008; revised October 27, 2008, January 09, 2009 and January 20, 2009. First published May 27, 2009; current version published June 10, 2009. This work was supported in part by the NSF CAREER Award CMS-0547448, AFOSR Contracts F49620-03-1-0381 and F49620-03-10170, AFRL Contract FA4819-05-D-0011, and the startup fund at Kansas State University. Recommended by Associate Editor A. Astolfi.

$\mathrm{G}$. Hu is with the Department of Mechanical and Nuclear Engineering, Kansas State University, Manhattan, KS 66506 (e-mail: gqhu@ksu.edu).

W. MacKunis, N. Gans, and W. E. Dixon are with the Department of Mechanical and Aerospace Engineering, University of Florida, Gainesville, FL 32611 USA (e-mail: mackunis@ufl.edu;ngans@ufl.edu;wdixon@ufl.edu).

J. Chen is with the Department of Naval Architecture and Marine Engineering, University of Michigan, Ann Arbor, MI 48109 USA (e-mail: jian.chen@ieee.org).

A. Behal is with the Department of Electrical and Computer Engineering, University of Central Florida, Orlando, FL 32809 USA (e-mail: abehal@mail. ucf.edu).

D. Dawson is with the Department of Electrical and Computer Engineering, Clemson University, Clemson, SC 29631 USA (e-mail: darren.dawson@ces. clemson.edu).

Digital Object Identifier 10.1109/TAC.2009.2015541 
Newton-step visual servo strategy with a Broyden Jacobian estimation for online singularity detection and avoidance in an uncalibrated visual servo control problem. In [4], [5] a recursive least-squares algorithm is implemented for Jacobian estimation, and a dynamic Gauss-Newton method is used to minimize the squared error in the image plane.

Robust control approaches based on static best-guess estimation of the calibration matrix have been developed to solve the uncalibrated visual servo regulation problem (cf. [9], [16]-[18]). Specifically, under a set of assumptions on the rotation and calibration matrix, a kinematic controller was developed in [9] that utilizes a constant, best-guess estimate of the calibration parameters to achieve local set-point regulation for the six degree-of-freedom (DOF) visual servo control problem. Homography-based visual servoing methods using best-guess estimation are used in [16]-[18] to achieve asymptotic or exponential regulation with respect to both camera and hand-eye calibration errors for the six DOF problem.

The development of traditional adaptive control methods to compensate for uncertainty in the camera calibration matrix is inhibited because of the time-varying uncertainty injected in the transformation from the normalization of the Euclidean coordinates. As a result, initial adaptive control results such as [6]-[12] were limited to scenarios where the optic axis of the camera was assumed to be perpendicular with the plane formed by the feature points (i.e., the time-varying uncertainty is reduced to a constant uncertainty) or assumed an additional sensor (e.g., ultrasonic sensors, laser-based sensors, additional cameras) could be used to measure the depth information.

More recent approaches exploit geometric relationships between multiple spatiotemporal views of an object to transform the time-varying uncertainty into known time-varying terms multiplied by an unknown constant [13], [14], [16]-[19]. In [13], an on-line calibration algorithm was developed for position-based visual servoing. In [14], an adaptive image-based visual servo controller was developed that regulated the feature points in an image to desired locations. One problem with methods based on the image-Jacobian is that the estimated image-Jacobian may contain singularities. The development in [14] exploits an additional potential force function to drive the estimated parameters away from the values that result in a singular Jacobian matrix. In [19], an adaptive homography-based controller was proposed to address problems of uncertainty in the intrinsic camera calibration parameters and lack of depth measurements. Specifically, an adaptive control strategy was developed from a Lyapunov-based approach that exploits the triangular structure of the calibration matrix. To the best of our knowledge, the result in [19] was the first result that regulates the robot end-effector to a desired position/orientation through visual servoing by actively compensating for the lack of depth measurements and uncertainty in the camera intrinsic calibration matrix with regard to the six DOF regulation problem. However, the relationship between the estimated rotation axis and the actual rotation axis is not correctly developed. A time-varying scaling factor was omitted which is required to relate the estimated rotation matrix and the actual rotation matrix. Specifically, the estimated rotation matrix and the actual rotation matrix were incorrectly related through eigenvectors that are associated with the eigenvalue of 1 . An unknown time-varying scalar is required to relate these vectors, and the methods in [19] do not appear to be suitable to accommodate for this uncertainty.

A new robust adaptive visual servo controller is developed in this technical note (and in the preliminary results in [18]) to asymptotically regulate the feature points in an image to the desired feature point locations while also regulating the six DOF position and orientation of the camera. These dual objectives are achieved by using a homography-based approach that exploits both image-space and reconstructed Euclidean information in the feedback loop. In comparison to pure

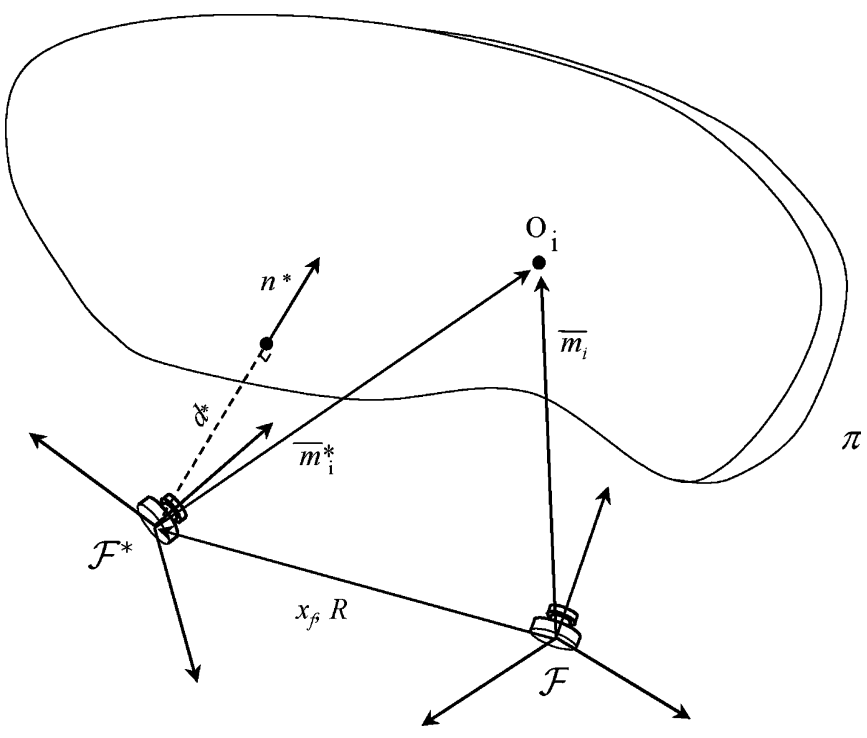

Fig. 1. Coordinate frame relationships between a camera viewing a planar patch at different spatiotemporal instances.

image-based feedback approaches, some advantages of using a homography-based method include: realizable Euclidean camera trajectories (see [20] and [21] for a discussion of Chaumette's Conundrum); a nonsingular image-Jacobian; and both the camera position and orientation and the feature point coordinates are included in the error system. Since some image-space information is used in the feedback-loop of the developed homography-based controller, the controller directly penalizes the system based on the feature point locations in comparison with pure position-based approaches. The developed controller is composed of the same adaptive translation controller as in the preliminary results in [19] and a new robust rotation controller. The contribution of the result is the development of the robust angular velocity controller that accommodates for the time-varying uncertain scaling factor by exploiting the upper triangular form of the rotation error system and the fact that the diagonal elements of the camera calibration matrix are positive.

\section{CAMERA Model AND EuCLIDEAn ReCONSTRUCTION}

\section{A. Camera Geometry}

Without loss of generality, ${ }^{1}$ the subsequent development is based on the assumption that four stationary coplanar and non-collinear feature points [24] denoted by $O_{i} \forall i=1,2,3,4$ can be determined from a feature point tracking algorithm. The plane defined by the four feature points is denoted by $\pi$ as depicted in Fig. 1. A coordinate frame $\mathcal{F}$ is considered to be affixed to the single current camera viewing the object, and a stationary coordinate frame $\mathcal{F}^{*}$ denotes a constant (a priori determined) desired camera position and orientation that is defined by a desired image. The Euclidean coordinates of the feature points $O_{i}$ expressed in the frames $\mathcal{F}$ and $\mathcal{F}^{*}$ are denoted by $x_{i}(t), y_{i}(t), z_{i}(t) \in \mathbb{R}$ and $x_{i}^{*}, y_{i}^{*}, z_{i}^{*} \in \mathbb{R}$, respectively. The normalized Euclidean coordinate vectors, denoted by $m_{i}(t) \in \mathbb{R}^{3}$ and $m_{i}^{*} \in \mathbb{R}^{3}$, are defined as

$$
m_{i} \triangleq\left[\begin{array}{lll}
\frac{x_{i}}{z_{i}} & \frac{y_{i}}{z_{i}} & 1
\end{array}\right]^{T} \quad m_{i}^{*} \triangleq\left[\begin{array}{lll}
\frac{x_{i}^{*}}{z_{i}^{*}} & \frac{y_{i}^{*}}{z_{i}^{*}} & 1
\end{array}\right]^{T}
$$

in $\mathcal{F}$ and $\mathcal{F}^{*}$, respectively.

\footnotetext{
${ }^{1}$ Image processing techniques can be used to select coplanar and non-collinear feature points within an image. However, if four coplanar target points are not available then the subsequent development can also exploit the virtual parallax algorithm (cf. [22], [23]) with no four of the eight target points being coplanar.
} 
From standard Euclidean geometry, relationships between $m_{i}(t)$ and $m_{i}^{*}$ can be determined as [24]

$$
m_{i}=\underbrace{\frac{z_{i}^{*}}{z_{i}}}_{\alpha_{i}} \underbrace{\left(R+\frac{x_{f}}{d^{*}} n^{* T}\right) m_{i}^{*}}_{H}
$$

where $\alpha_{i}(t) \in \mathbb{R}$ is a scaling term, and $H(t) \in \mathbb{R}^{3 \times 3}$ denotes the Euclidean homography. The Euclidean homography is composed of a scaled translation vector which is equal to the translation vector $x_{f}(t) \in \mathbb{R}^{3}$ divided by the distance $d^{*} \in \mathbb{R}$ from the origin of $\mathcal{F}^{*}$ to the plane $\pi$, the rotation between $\mathcal{F}$ and $\mathcal{F}^{*}$ denoted by $R(t) \in S O(3)$, and $n^{*} \in \mathbb{R}^{3}$ denoting a constant unit normal to the plane $\pi$.

Each feature point $O_{i}$ on $\pi$ also has a pixel coordinate $p_{i}(t) \in \mathbb{R}^{3}$ and $p_{i}^{*} \in \mathbb{R}^{3}$ expressed in the image coordinate frame for the current image and the desired image denoted by

$$
p_{i} \triangleq\left[\begin{array}{lll}
u_{i} & v_{i} & 1
\end{array}\right]^{T} \quad p_{i}^{*} \triangleq\left[\begin{array}{lll}
u_{i}^{*} & v_{i}^{*} & 1
\end{array}\right]^{T}
$$

where $u_{i}(t), v_{i}(t), u_{i}^{*}, v_{i}^{*} \in \mathbb{R}$. The pixel coordinates $p_{i}(t)$ and $p_{i}^{*}$ are related to the normalized task-space coordinates $m_{i}(t)$ and $m_{i}^{*}$ by the following global invertible transformation (i.e., the pinhole camera model)

$$
p_{i}=A m_{i} \quad p_{i}^{*}=A m_{i}^{*}
$$

where $A \in \mathbb{R}^{3 \times 3}$ is a constant, upper triangular, and invertible intrinsic camera calibration matrix that is explicitly defined as [25]

$$
A \triangleq\left[\begin{array}{ccc}
\alpha & -\alpha \cot \phi & u_{0} \\
0 & \frac{\beta}{\sin \phi} & v_{0} \\
0 & 0 & 1
\end{array}\right]=\left[\begin{array}{ccc}
a_{11} & a_{12} & a_{13} \\
0 & a_{22} & a_{23} \\
0 & 0 & 1
\end{array}\right] .
$$

In (5), $u_{0}, v_{0} \in \mathbb{R}$ denote the pixel coordinates of the principal point (i.e., the image center that is defined as the frame buffer coordinates of the intersection of the optical axis with the image plane), $\alpha, \beta \in \mathbb{R}$ represent the product of the camera scaling factors and the focal length, and $\phi \in \mathbb{R}$ is the skew angle between the camera axes. Based on the physical meaning, the diagonal calibration elements are positive (i.e., $a_{11}, a_{22}>0$ ).

Assumption 1: The bounds of $a_{11}$ and $a_{22}$ are assumed to be known as

$$
\underline{\zeta}_{a_{11}}<a_{11}<\bar{\zeta}_{a_{11}} \quad \underline{\zeta}_{a_{22}}<a_{22}<\bar{\zeta}_{a_{22}} .
$$

The absolute values of $a_{12}, a_{13}, a_{23}$ are upper bounded as

$$
\left|a_{12}\right|<\bar{\zeta}_{a_{12}} \quad\left|a_{13}\right|<\bar{\zeta}_{a_{13}} \quad\left|a_{23}\right|<\bar{\zeta}_{a_{23}} .
$$

In (6) and (7), $\underline{\zeta}_{a_{11}}, \bar{\zeta}_{a_{11}}, \underline{\zeta}_{a_{22}}, \bar{\zeta}_{a_{22}}, \bar{\zeta}_{a_{12}}, \bar{\zeta}_{a_{13}}$ and $\bar{\zeta}_{a_{23}}$ are known positive constants.

Assumption 2: The reference plane is within the camera's field of view and not at infinity. That is, there exist positive constants $\underline{\zeta}_{z_{i}}$ and $\bar{\zeta}_{z_{i}}$ such that

$$
\underline{\zeta}_{z_{i}}<z_{i}(t)<\bar{\zeta}_{z_{i}}
$$

\section{B. Reconstruction Using Vanishing Points}

The subsequent development exploits the use of vanishing points to relax the requirement that the camera is perfectly calibrated. This assumption limits the application of the subsequent methods to environments where vanishing points can be detected in an image (see [26] for a description of how to determine vanishing points in an image). However, vanishing points can be determined from parallel lines that are present in most indoor environments, or for systems navigating in an urban environment, road following, and etc.
Based on (2)-(4), the homography relationship based on measurable pixel coordinates is:

$$
p_{i}=\alpha_{i} A H A^{-1} p_{i}^{*} .
$$

Since $A$ is unknown, standard homography computation and decomposition algorithms can't be applied to extract the rotation and translation from the homography. As stated in [16], if some additional information is known, such as four vanishing points, the rotation matrix can be obtained. Projective transformations map infinite points to finite points. That is, when a Euclidean point approaches a point at infinity, its image projection (i.e., a vanishing point) is mapped to a finite point. Hence, a homography can be developed to map four non-colinear Euclidean points on a plane at infinity (i.e., $d^{*}=\infty$ ) to the retinal plane of the camera. ${ }^{2}$ The vanishing point homography depends only on the relative orientation and the intrinsic camera calibration parameters as

$$
H=R+\frac{x_{f}}{d^{*}} n^{* T}=R .
$$

Based on (10), the relationship in (9) can be expressed as

$$
p_{i}=\alpha_{i} \bar{R} p_{i}^{*}
$$

where $\bar{R}(t) \in \mathbb{R}^{3 \times 3}$ is defined as

$$
\bar{R}=A R A^{-1} .
$$

For the four vanishing points, twelve linear equations can be obtained based on (11). After normalizing $\bar{R}(t)$ by one nonzero element (e.g., $\bar{R}_{33}(t) \in \mathbb{R}$ which is assumed to be the third row third column element of $\bar{R}(t)$ without loss of generality) twelve equations can be used to solve for twelve unknowns. The twelve unknowns are given by the eight unknown elements of the normalized $\bar{R}(t)$, denoted by $\bar{R}_{n}(t) \in \mathbb{R}^{3 \times 3}$ defined as

$$
\bar{R}_{n} \triangleq \frac{\bar{R}}{\bar{R}_{33}}
$$

and the four unknowns are given by $\bar{R}_{33}(t) \alpha_{i}(t)$ for $i=1,2,3,4$. From the definition of $\bar{R}_{n}(t)$ in (13), the fact that

$$
\operatorname{det}(\bar{R})=\operatorname{det}(A) \operatorname{det}(R) \operatorname{det}\left(A^{-1}\right)=1
$$

can be used to conclude that

$$
\bar{R}_{33}^{3} \operatorname{det}\left(\bar{R}_{n}\right)=1
$$

and hence,

$$
\bar{R}=\frac{\bar{R}_{n}}{\sqrt[3]{\operatorname{det}\left(\bar{R}_{n}\right)}} .
$$

After $\bar{R}(t)$ is obtained, the original four featurepoints on the reference plane can be used to determine the depth ratio $\alpha_{i}(t)$.

\section{OPEN-LOOP ERROR SYSTEM}

\section{A. Rotation Error System}

If the rotation matrix $R(t)$ introduced in (2) were known, then the corresponding unit quaternion $q(t) \triangleq\left[q_{0}(t) q_{v}^{T}(t)\right]^{T}$ can be calculated using the numerically robust method presented in [28] and [29] based on the corresponding relationships

$$
R(q)=\left(q_{0}^{2}-q_{v}^{T} q_{v}\right) I_{3}+2 q_{v} q_{v}^{T}-2 q_{0} q_{v}^{\times}
$$

${ }^{2} \mathrm{An}$ in-depth discussion related to the vanishing point homography is provided in Chapter 1 of [25], [27]. 
where $q_{0}(t) \in \mathbb{R}, q_{v}(t) \in \mathbb{R}^{3}, I_{3}$ is the $3 \times 3$ identity matrix, and the notation $q_{v}^{\times}(t)$ denotes the following skew-symmetric form of the vector $q_{v}(t)$ :

$$
q_{v}^{\times}=\left[\begin{array}{ccc}
0 & -q_{v 3} & q_{v 2} \\
q_{v 3} & 0 & -q_{v 1} \\
-q_{v 2} & q_{v 1} & 0
\end{array}\right], \forall q_{v}=\left[\begin{array}{l}
q_{v 1} \\
q_{v 2} \\
q_{v 3}
\end{array}\right] .
$$

Given $R(t)$, the quaternion $q(t)$ can also be written as

$$
\begin{aligned}
& q_{0}=\frac{1}{2} \sqrt{1+\operatorname{tr}(R)} \\
& q_{v}=\frac{1}{2} u \sqrt{3-\operatorname{tr}(R)}
\end{aligned}
$$

where $u(t) \in \mathbb{R}^{3}$ is a unit eigenvector of $R(t)$ with respect to the eigenvalue 1 .

To develop the open-loop rotation error system, we first exploit the fact that any two unit quaternions can be related by some incremental transition quaternion. Specifically, $q(t)$ and $q(t+\triangle t)$ can be related by $\triangle q(t)$ as

$$
q(t+\triangle t)=q(t) \triangle q(t)
$$

where the quaternion $\triangle q(t)$ is written as

$$
\triangle q(t)=\left[\begin{array}{c}
\cos \left(\frac{\triangle \theta}{2}\right) \\
u_{c} \sin \left(\frac{\triangle \theta}{2}\right)
\end{array}\right], u_{c} \in \mathbb{R}^{3}, \triangle \theta \in \mathbb{R}
$$

which can be approximated as follows for incremental motion [30]:

$$
\triangle q(t) \approx\left[\begin{array}{c}
1 \\
u_{c} \frac{\Delta \theta}{2}
\end{array}\right]
$$

In (21) and (22), $u_{c}(t)$ denotes the unit vector rotation axis, and $\triangle \theta(t)$ denotes the rotation angle. Multiplication for two quaternions $q(t)=$ $\left[\begin{array}{llll}q_{0} & q_{v 1} & q_{v 2} & q_{v 3}\end{array}\right]^{T}$ and $p(t)=\left[\begin{array}{llll}p_{0} & p_{v 1} & p_{v 2} & p_{v 3}\end{array}\right]^{T}$ are defined as

$$
q(t) p(t)=\left[\begin{array}{cccc}
q_{0} & -q_{v 1} & -q_{v 2} & -q_{v 3} \\
q_{v 1} & q_{0} & -q_{v 3} & q_{v 2} \\
q_{v 2} & q_{v 3} & q_{0} & -q_{v 1} \\
q_{v 3} & -q_{v 2} & q_{v 1} & q_{0}
\end{array}\right]\left[\begin{array}{c}
p_{0} \\
p_{v 1} \\
p_{v 2} \\
p_{v 3}
\end{array}\right] .
$$

From the previous development, (20) can be expressed as

$$
\begin{aligned}
q(t+\triangle t) & =q(t)\left[\begin{array}{c}
1 \\
u_{c} \frac{\triangle \theta}{2}
\end{array}\right] \\
& =q(t)+q(t)\left[\begin{array}{c}
0 \\
u_{c} \frac{\triangle \theta}{2}
\end{array}\right] .
\end{aligned}
$$

Based on (24), the time derivative of the incremental change in the quaternion can be expressed as

$$
\frac{q(t+\triangle t)-q(t)}{\triangle t}=q(t)\left[\begin{array}{c}
0 \\
u_{c} \frac{\triangle \theta}{2 \triangle t}
\end{array}\right]=q(t)\left[\begin{array}{c}
0 \\
\frac{\omega_{c}}{2}
\end{array}\right]
$$

where $\omega_{c}(t) \in \mathbb{R}^{3}$ defines the angular velocity of the camera expressed in $\mathcal{F}$. By using the multiplication rule in (23), the open-loop error system in (25) can be expressed as [31]

$$
\left[\begin{array}{c}
\dot{q}_{0} \\
\dot{q}_{v}
\end{array}\right]=\frac{1}{2}\left[\begin{array}{c}
-q_{v}^{T} \\
q_{0} I_{3}+q_{v}^{\times}
\end{array}\right] \omega_{c} .
$$

The quaternion $q(t)$ given in (17)-(26) is not measurable since $R(t)$ is unknown. However, since $\bar{R}(t)$ can be determined as described in (16), the same algorithm as shown in equations (18) and (19) can be used to determine a corresponding measurable quaternion $\left(\bar{q}_{0}(t), \bar{q}_{v}^{T}(t)\right)^{T}$ as

$$
\begin{aligned}
& \bar{q}_{0}=\frac{1}{2} \sqrt{1+\operatorname{tr}(\bar{R})} \\
& \bar{q}_{v}=\frac{1}{2} \bar{u} \sqrt{3-\operatorname{tr}(\bar{R})}
\end{aligned}
$$

where $\bar{u}(t) \in \mathbb{R}^{3}$ is a unit eigenvector of $\bar{R}(t)$ with respect to the eigenvalue 1 . The rotation axis $\bar{u}(t)$ has two solutions. Either solution will generate the same regulation result provided the different rotation directions (clockwise or counterclockwise) are assigned in a consistent manner.

Based on (12), $\operatorname{tr}(\bar{R})=\operatorname{tr}\left(A R A^{-1}\right)=\operatorname{tr}(R)$, where $\operatorname{tr}(\cdot)$ denotes the trace of a matrix. Since $R(t)$ and $\bar{R}(t)$ are similar matrices, the relationship between $\left(q_{0}(t), q_{v}^{T}(t)\right)^{T}$ and $\left(\bar{q}_{0}(t), \bar{q}_{v}^{T}(t)\right)^{T}$ can be determined as

$$
\bar{q}_{0}=q_{0} \quad \bar{q}_{v}=\frac{\left\|q_{v}\right\|}{\left\|A q_{v}\right\|} A q_{v} \triangleq \gamma A q_{v}
$$

where $\gamma(t) \in \mathbb{R}$ is a positive, unknown, time-varying scalar that satisfies the following inequalities (see Appendix)

$$
\underline{\zeta}_{\gamma}<\gamma(t)<\bar{\zeta}_{\gamma}
$$

where $\underline{\zeta}_{\gamma}, \bar{\zeta}_{\gamma} \in \mathbb{R}$ are positive bounding constants. The inverse of the relationship between $\bar{q}_{v}(t)$ and $q_{v}(t)$ in (29) can be developed as

$$
\begin{aligned}
q_{v} & =\frac{1}{\gamma} A^{-1} \bar{q}_{v} \\
& =\frac{1}{\gamma}\left[\begin{array}{c}
\frac{1}{a_{11}} \bar{q}_{v 1}-\frac{a_{12}}{a_{11} a_{22}} \bar{q}_{v 2}-\left(\frac{a_{13}}{a_{11}}-\frac{a_{12} a_{23}}{a_{11} a_{22}}\right) \bar{q}_{v 3} \\
\frac{1}{a_{22}} \bar{q}_{v 2}-\frac{a_{23}}{a_{22}} \bar{q}_{v 3} \\
\bar{q}_{v 3}
\end{array}\right] .
\end{aligned}
$$

\section{B. Translation Error System}

The translation error, denoted by $e(t) \in \mathbb{R}^{3}$, is defined as

$$
e(t)=p_{e}(t)-p_{e}^{*}
$$

where $p_{e}(t), p_{e}^{*} \in \mathbb{R}^{3}$ are

$$
p_{e}=\left[\begin{array}{lll}
u_{i} & v_{i} & -\ln \left(\alpha_{i}\right)
\end{array}\right]^{T} \quad p_{e}^{*}=\left[\begin{array}{lll}
u_{i}^{*} & v_{i}^{*} & 0
\end{array}\right]^{T}
$$

where $i \in\{1, \cdots, 4\}$. The translation error $e(t)$ is measurable since the first two elements are image coordinates, and $\alpha_{i}(t)$ is obtained from the homography decomposition. The open-loop translation error system can be obtained by taking the time derivative of $e(t)$ and multiplying the resulting expression by $z_{i}^{*}$ as

$$
z_{i}^{*} \dot{e}=-\alpha_{i} A_{e} v_{c}+z_{i}^{*} A_{e}\left(A^{-1} p_{i}\right)^{\times} \omega_{c}
$$

where $v_{c}(t) \in \mathbb{R}^{3}$ defines the linear velocity of the camera expressed in $\mathcal{F}$, and $A_{e}(t) \in \mathbb{R}^{3 \times 3}$ is defined as

$$
A_{e}=\left[\begin{array}{ccc}
a_{11} & a_{12} & a_{13}-u_{i} \\
0 & a_{22} & a_{23}-v_{i} \\
0 & 0 & 1
\end{array}\right]
$$


To facilitate the control development, the translation error system can be linearly parameterized as

$$
\begin{array}{r}
z_{i}^{*}\left[\begin{array}{c}
\dot{e}_{1} \\
\dot{e}_{2} \\
\dot{e}_{3}
\end{array}\right]=-\alpha_{i}\left[\begin{array}{c}
a_{11} v_{c 1}+a_{12} v_{c 2}+v_{c 3}\left(a_{13}-u_{i}\right) \\
a_{22} v_{c 2}+v_{c 3}\left(a_{23}-v_{i}\right) \\
v_{c 3}
\end{array}\right] \\
+z_{i}^{*}\left[\begin{array}{l}
\bar{Y}_{1}\left(u_{i}, v_{i}, \omega_{c}\right) \bar{\phi} \\
\bar{Y}_{2}\left(u_{i}, v_{i}, \omega_{c}\right) \bar{\phi} \\
\bar{Y}_{3}\left(u_{i}, v_{i}, \omega_{c}\right) \bar{\phi}
\end{array}\right]
\end{array}
$$

where $\bar{Y}_{i}(\cdot) \in \mathbb{R}^{1 \times m}, i=1,2,3$, are known regressor vectors that do not depend on the calibration parameters, and $\bar{\phi} \in \mathbb{R}^{m}$ is a vector of constant unknown parameters.

\section{Control DEVElopment}

For generality, the subsequent control development is based on the assumption that the control inputs are the linear and angular velocity of the camera. For some applications (e.g., the camera is held by a robot manipulator), the control input may need to be transformed into a different space (e.g., the joint-space of the robot) where potential singularities could arise in the transformation (e.g., robot Jacobian). Also, the kinematics/dynamics may include motion constraints (e.g., nonholonomic constraints of a mobile vehicle) that may need to be considered.

\section{A. Rotation Control Development and Stability Analysis}

Based on the relationship in (29), the open-loop error system in (26), and the subsequent stability analysis, the rotation controller is designed as

$$
\begin{aligned}
& \omega_{c 1}=-k_{\omega 1} \bar{q}_{v 1}=-\left(k_{\omega 11}+2\right) \bar{q}_{v 1} \\
& \omega_{c 2}=-k_{\omega 2} \bar{q}_{v 2}=-\left(k_{\omega 21}+k_{\omega 22}+1\right) \bar{q}_{v 2} \\
& \omega_{c 3}=-k_{\omega 3} \bar{q}_{v 3}=-\left(k_{\omega 31}+k_{\omega 32}+k_{\omega 33}\right) \bar{q}_{v 3}
\end{aligned}
$$

where $k_{\omega i} \in \mathbb{R}, i=1,2,3$ and $k_{\omega i j} \in \mathbb{R}, i, j=1,2,3, j<i$, are positive constants. The expressed form of the controller in (36) is motivated by the use of completing the squares in the subsequent stability analysis. In (36), the damping control gains $k_{\omega 21}, k_{\omega 31}, k_{\omega 32}$ are selected according to the following sufficient conditions to facilitate the subsequent stability analysis

$$
\begin{aligned}
& k_{\omega 21}>\frac{1}{4} k_{\omega 1}^{2} \frac{\bar{\zeta}_{a_{12}}^{2}}{\underline{\zeta}_{a_{11}} \underline{\zeta}_{a_{22}}} \\
& k_{\omega 31}>\frac{1}{4} k_{\omega 1}^{2} \frac{1}{\underline{\zeta}_{a_{11}}}\left(\frac{\bar{\zeta}_{a_{12}} \bar{\zeta}_{a_{23}}}{\underline{\zeta}_{a_{22}}}+\bar{\zeta}_{a_{13}}\right)^{2} \\
& k_{\omega 32}>\frac{1}{4} k_{\omega 2}^{2} \frac{\bar{\zeta}_{a_{23}}^{2}}{\underline{\zeta}_{a_{22}}}
\end{aligned}
$$

where $\underline{\zeta}_{a_{11}}, \bar{\zeta}_{a_{11}}, \underline{\zeta}_{a_{22}}, \bar{\zeta}_{a_{22}}, \bar{\zeta}_{a_{12}}, \bar{\zeta}_{a_{13}}$ and $\bar{\zeta}_{a_{23}}$ are defined in (6) and (7), and $k_{\omega 11}, k_{\omega 22}, k_{\omega 33}$ are feedback gains that can be selected to adjust the performance of the rotation control system.

Proposition 1: Provided the sufficient gain conditions given in (37) are satisfied, the controller in (36) ensures asymptotic regulation of the rotation error in the sense that

$$
\left\|q_{v}(t)\right\| \rightarrow 0, \quad \text { as } \quad t \rightarrow \infty .
$$

Proof: Let $V_{1}\left(q_{v}, q_{0}\right) \in \mathbb{R}$ denote the following non-negative function:

$$
V_{1} \triangleq q_{v}^{T} q_{v}+\left(1-q_{0}\right)^{2}
$$

Based on the open-loop error system in (26), the time-derivative of $V_{1}(t)$ can be determined as

$$
\dot{V}_{1}=2 q_{v}^{T} \dot{q}_{v}-2\left(1-q_{0}\right) \dot{q}_{0}=q_{v}^{T} \omega_{c}=q_{v 1} \omega_{c 1}+q_{v 2} \omega_{c 2}+q_{v 3} \omega_{c 3} .
$$

After substituting (31) for $q_{v}(t)$ and substituting (36) for $\omega_{c}(t)$, the expression in (40) can be simplified as

$$
\begin{aligned}
& \gamma \dot{V}_{1}=-\left(k_{\omega 11} \frac{1}{a_{11}} \bar{q}_{v 1}^{2}+k_{\omega 22} \frac{1}{a_{22}} \bar{q}_{v 2}^{2}+k_{\omega 33} \bar{q}_{v 3}^{2}\right) \\
& -\frac{1}{a_{11}}\left[\bar{q}_{v 1}^{2}-k_{\omega 1} \frac{a_{12}}{a_{22}} \bar{q}_{v 1} \bar{q}_{v 2}+k_{\omega 21} \frac{a_{11}}{a_{22}} \bar{q}_{v 2}^{2}\right] \\
& -\frac{1}{a_{11}}\left[\bar{q}_{v 1}^{2}+k_{\omega 1}\left(\frac{a_{12} a_{23}}{a_{22}}-a_{13}\right) \bar{q}_{v 1} \bar{q}_{v 3}\right. \\
& \left.+k_{\omega 31} a_{11} \bar{q}_{v 3}^{2}\right] \\
& -\frac{1}{a_{22}}\left[\bar{q}_{v 2}^{2}-k_{\omega 2} a_{23} \bar{q}_{v 2} \bar{q}_{v 3}+k_{\omega 32} a_{22} \bar{q}_{v 3}^{2}\right] \text {. }
\end{aligned}
$$

After completing the squares on each of the bracketed terms in (41), the expression in (41) can be written as

$$
\begin{aligned}
& \gamma \dot{V}_{1}=-\left(k_{\omega 11} \frac{1}{a_{11}} \bar{q}_{v 1}^{2}+k_{\omega 22} \frac{1}{a_{22}} \bar{q}_{v 2}^{2}+k_{\omega 33} \bar{q}_{v 3}^{2}\right) \\
&-\frac{1}{a_{11}}\left[\left(\bar{q}_{v 1}-k_{\omega 1} \frac{a_{12}}{2 a_{22}} \bar{q}_{v 2}\right)^{2}\right.\left.+\frac{a_{11}}{a_{22}}\left(k_{\omega 21}-\frac{1}{4} k_{\omega 1}^{2} \frac{a_{12}^{2}}{a_{11} a_{22}}\right) \bar{q}_{v 2}^{2}\right] \\
&-\frac{1}{a_{11}}\left[\left(\bar{q}_{v 1}+\frac{1}{2} k_{\omega 1}\left(\frac{a_{12} a_{23}}{a_{22}}-a_{13}\right) \bar{q}_{v 3}\right)^{2}\right. \\
&\left.+a_{11}\left(k_{\omega 31}-\frac{1}{4} k_{\omega 1}^{2} \frac{1}{a_{11}}\left(\frac{a_{12} a_{23}}{a_{22}}-a_{13}\right)^{2}\right) \bar{q}_{v 3}^{2}\right] \\
&-\frac{1}{a_{22}}\left[\left(\bar{q}_{v 2}-\frac{1}{2} k_{\omega 2} a_{23} \bar{q}_{v 3}\right)^{2}\right. \\
&\left.+a_{22}\left(k_{\omega 32}-\frac{1}{4} k_{\omega 2}^{2} \frac{a_{23}^{2}}{a_{22}}\right) \bar{q}_{v 3}^{2}\right] .
\end{aligned}
$$

Provided the sufficient gain conditions given in (37) are satisfied, then (42) can be upper bounded as

$$
\gamma \dot{V}_{1}<-\left(k_{\omega 11} \frac{1}{a_{11}} \bar{q}_{v 1}^{2}+k_{\omega 22} \frac{1}{a_{22}} \bar{q}_{v 2}^{2}+k_{\omega 33} \bar{q}_{v 3}^{2}\right) .
$$

Based on (30), the inequality in (43) can be further upper bounded as

$$
\dot{V}_{1}<-\frac{1}{\bar{\zeta}_{\gamma}}\left(k_{\omega 11} \frac{1}{a_{11}} \bar{q}_{v 1}^{2}+k_{\omega 22} \frac{1}{a_{22}} \bar{q}_{v 2}^{2}+k_{\omega 33} \bar{q}_{v 3}^{2}\right) .
$$

The Lyapunov function given in (39) and its time derivative in (44) can be used to conclude that $q_{v}(t), q_{0}(t) \in \mathcal{L}_{\infty}$ and $\bar{q}_{v}(t) \in \mathcal{L}_{2}$ (of course, $q_{v}(t), q_{0}(t) \in \mathcal{L}_{\infty}$ by definition also). The expressions in (29) and (31), and the fact that $\bar{q}_{v}(t) \in \mathcal{L}_{2}$, can be used to conclude that $q_{v}(t) \in \mathcal{L}_{2}$. Since $q_{v}(t), q_{0}(t) \in \mathcal{L}_{\infty}$, then $R(t), \bar{R}(t), \bar{q}_{v}(t)$ and $\bar{q}_{0}(t) \in \mathcal{L}_{\infty}$. Hence, (36) can be used to conclude that $\omega_{c}(t) \in \mathcal{L}_{\infty}$. Based on the rotation error system in $(26), \dot{q}_{v}(t), \dot{q}_{0}(t) \in \mathcal{L}_{\infty}$; hence, $q_{v}(t), q_{0}(t)$ are uniformly continuous. Barbalat's lemma can now be used to conclude that $\left\|q_{v}(t)\right\| \rightarrow 0$ as $t \rightarrow \infty$. 


\section{B. Translation Control Development and Stability Analysis}

After some algebraic manipulation, the translation error system in (35) can be rewritten as

$$
\begin{aligned}
\frac{z_{i}^{*}}{a_{11}} \dot{e}_{1} & =-\alpha_{i} v_{c 1}+Y_{1}\left(\alpha_{i}, u_{i}, v_{i}, \omega_{c}, v_{c 2}, v_{c 3}\right) \phi_{1} \\
\frac{z_{i}^{*}}{a_{22}} \dot{e}_{2} & =-\alpha_{i} v_{c 2}+Y_{2}\left(\alpha_{i}, u_{i}, v_{i}, \omega_{c}, v_{c 3}\right) \phi_{2} \\
z_{i}^{*} \dot{e}_{3} & =-\alpha_{i} v_{c 3}+Y_{3}\left(\alpha_{i}, u_{i}, v_{i}, \omega_{c}\right) \phi_{3}
\end{aligned}
$$

where $\phi_{1} \in \mathbb{R}^{n_{1}}, \phi_{2} \in \mathbb{R}^{n_{2}}$, and $\phi_{3} \in \mathbb{R}^{n_{3}}$ are vectors of constant unknown parameters, and the known regressor vectors $Y_{1}(\cdot) \in \mathbb{R}^{1 \times n_{1}}$, $Y_{2}(\cdot) \in \mathbb{R}^{1 \times n_{2}}$, and $Y_{3}(\cdot) \in \mathbb{R}^{1 \times n_{3}}$ satisfy the following equations:

$$
\begin{aligned}
Y_{1} \phi_{1} & =-\alpha_{i} \frac{a_{12}}{a_{11}} v_{c 2}-\alpha_{i} \frac{\left(a_{13}-u_{i}\right)}{a_{11}} v_{c 3}+z_{i}^{*} \bar{Y}_{1}\left(u_{i}, v_{i}, \omega_{c}\right) \frac{\bar{\phi}}{a_{11}} \\
Y_{2} \phi_{2} & =-\alpha_{i} \frac{a_{23}-v_{i}}{a_{22}} v_{c 3}+z_{i}^{*} \bar{Y}_{2}\left(u_{i}, v_{i}, \omega_{c}\right) \frac{\bar{\phi}}{a_{22}} \\
Y_{3} \phi_{3} & =z_{i}^{*} \bar{Y}_{3}\left(u_{i}, v_{i}, \omega_{c}\right) \bar{\phi} .
\end{aligned}
$$

The control strategy is to design $v_{c 3}(t)$ to stabilize $e_{3}(t)$, and then design $v_{c 2}(t)$ to stabilize $e_{2}(t)$ given $v_{c 3}(t)$, and then design $v_{c 1}(t)$ to stabilize $e_{1}(t)$ given $v_{c 3}(t)$ and $v_{c 2}(t)$. Following this design strategy, the translation controller $v_{c}(t)$ is designed as

$$
\begin{aligned}
& v_{c 3}=\frac{1}{\alpha_{i}}\left(k_{v 3} e_{3}+Y_{3}\left(\alpha_{i}, u_{i}, v_{i}, \omega_{c}\right) \hat{\phi}_{3}\right) \\
& v_{c 2}=\frac{1}{\alpha_{i}}\left(k_{v 2} e_{2}+Y_{2}\left(\alpha_{i}, u_{i}, v_{i}, \omega_{c}, v_{c 3}\right) \hat{\phi}_{2}\right) \\
& v_{c 1}=\frac{1}{\alpha_{i}}\left(k_{v 1} e_{1}+Y_{1}\left(\alpha_{i}, u_{i}, v_{i}, \omega_{c}, v_{c 2}, v_{c 3}\right) \hat{\phi}_{1}\right)
\end{aligned}
$$

where $k_{v i} \in \mathbb{R}, i=1,2,3$ are positive constants and the depth ratio $\alpha_{i}(t)>0 \forall t$. In (46), $\hat{\phi}_{1}(t) \in \mathbb{R}^{n_{1}}, \hat{\phi}_{2}(t) \in \mathbb{R}^{n_{2}}, \hat{\phi}_{3}(t) \in \mathbb{R}^{n_{3}}$ denote adaptive estimates that are designed according to the following adaptive update laws to cancel the respective terms in the subsequent stability analysis

$$
\dot{\hat{\phi}}_{1}=\Gamma_{1} Y_{1}^{T} e_{1} \quad \dot{\hat{\phi}}_{2}=\Gamma_{2} Y_{2}^{T} e_{2} \quad \dot{\hat{\phi}}_{3}=\Gamma_{3} Y_{3}^{T} e_{3}
$$

where $\Gamma_{1} \in \mathbb{R}^{n_{1} \times n_{1}}, \Gamma_{2} \in \mathbb{R}^{n_{2} \times n_{2}}, \Gamma_{3} \in \mathbb{R}^{n_{3} \times n_{3}}$ are diagonal matrices of positive constant adaptation gains. Based on (45) and (46), the closed-loop translation error system is

$$
\begin{aligned}
\frac{z_{i}^{*}}{a_{11}} \dot{e}_{1} & =-k_{v 1} e_{1}+Y_{1}\left(\alpha_{i}, u_{i}, v_{i}, \omega_{c}, v_{c 2}, v_{c 3}\right) \tilde{\phi}_{1} \\
\frac{z_{i}^{*}}{a_{22}} \dot{e}_{2} & =-k_{v 2} e_{2}+Y_{2}\left(\alpha_{i}, u_{i}, v_{i}, \omega_{c}, v_{c 3}\right) \tilde{\phi}_{2} \\
z_{i}^{*} \dot{e}_{3} & =-k_{v 3} e_{3}+Y_{3}\left(\alpha_{i}, u_{i}, v_{i}, \omega_{c}\right) \tilde{\phi}_{3}
\end{aligned}
$$

where $\tilde{\phi}_{1}(t) \in \mathbb{R}^{n_{1}}, \tilde{\phi}_{2}(t) \in \mathbb{R}^{n_{2}}, \tilde{\phi}_{3}(t) \in \mathbb{R}^{n_{3}}$ denote the intrinsic calibration parameter mismatch defined as

$$
\tilde{\phi}_{1}(t)=\phi_{1}-\hat{\phi}_{1}(t) \quad \tilde{\phi}_{2}(t)=\phi_{2}-\hat{\phi}_{2}(t) \quad \tilde{\phi}_{3}(t)=\phi_{3}-\hat{\phi}_{3}(t) .
$$

Proposition 2: The controller given in (46) along with the adaptive update law in (47) ensures asymptotic regulation of the translation error system in the sense that

$$
\|e(t)\| \rightarrow 0 \text { as } t \rightarrow \infty \text {. }
$$

Proof: Let $V_{2}\left(e, \tilde{\phi}_{1}, \tilde{\phi}_{2}, \tilde{\phi}_{3}\right) \in \mathbb{R}$ denote the following non-negative function:

$$
\begin{aligned}
V_{2}=\frac{1}{2} \frac{z_{i}^{*}}{a_{11}} e_{1}^{2}+\frac{1}{2} \frac{z_{i}^{*}}{a_{22}} e_{2}^{2}+\frac{1}{2} z_{i}^{*} e_{3}^{2}+\frac{1}{2} \tilde{\phi}_{1}^{T} \Gamma_{1}^{-1} \tilde{\phi}_{1} \\
+\frac{1}{2} \tilde{\phi}_{2}^{T} \Gamma_{2}^{-1} \tilde{\phi}_{2}+\frac{1}{2} \tilde{\phi}_{3}^{T} \Gamma_{3}^{-1} \tilde{\phi}_{3} .
\end{aligned}
$$

After taking the time derivative of (49) and substituting for the closedloop error system developed in (48), the following simplified expression can be obtained:

$$
\dot{V}_{2}=-k_{v 1} e_{1}^{2}-k_{v 2} e_{2}^{2}-k_{v 3} e_{3}^{2} .
$$

Based on (49) and (50), $e_{1}(t), e_{2}(t), e_{3}(t) \in \mathcal{L}_{\infty} \cap \mathcal{L}_{2}$, and $\tilde{\phi}_{1}(t), \hat{\phi}_{1}(t), \tilde{\phi}_{2}(t), \hat{\phi}_{2}(t), \tilde{\phi}_{3}(t), \hat{\phi}_{3}(t) \in \mathcal{L}_{\infty}$. Based on the assumption that $\underline{\zeta}_{z_{i}}<z_{i}(t)<\bar{\zeta}_{z_{i}}$, the expression in (46) can be used to conclude that $v_{c}(t) \in \mathcal{L}_{\infty}$. Based on the previous stability analysis for the rotation controller, $\omega_{c}(t) \in \mathcal{L}_{\infty}$; hence, (48) can be used to conclude that $\dot{e}_{1}(t), \dot{e}_{2}(t), \dot{e}_{3}(t) \in \mathcal{L}_{\infty}$ (i.e., $e_{1}(t), e_{2}(t), e_{3}(t)$ are uniformly continuous). Barbalat's lemma can now be used to show that $e_{1}(t), e_{2}(t), e_{3}(t) \rightarrow 0$ as $t \rightarrow \infty$.

Based on Propositions 1 and 2, the main result can be stated as follows.

Theorem 1: The controller given in (36) and (46) along with the adaptive update law in (47) ensures asymptotic translation and rotation regulation in the sense that

$$
\left\|q_{v}(t)\right\| \rightarrow 0 \text { and } \quad\|e(t)\| \rightarrow 0 \quad \text { as } \quad t \rightarrow \infty
$$

provided the control gains satisfy the sufficient conditions given in (37). Proof: See proofs in Propositions 1 and 2.

\section{CONCLUSION}

A robust adaptive visual servo controller is proposed to asymptotically regulate the robot end-effector to a desired pose despite uncertainty in the distance from the camera to the target and parametric uncertainty in the camera calibration matrix. A Lyapunov-based stability analysis was used to prove asymptotic stability for the six DOF homography-based visual servo controller. To achieve the result, a high-gain robust controller is developed to asymptotically stabilize the rotation error system, whereas, an adaptive controller is developed to stabilize the translation error by compensating for the unknown depth information and intrinsic camera calibration parameters. Future work will include experimental evaluation of the presented controller, including evaluating performance against similar controllers with well calibrated and poorly calibrated cameras.

\section{APPENDIX \\ INEQUALITY DEVELOPMENT}

Property: There exist two positive constants $\underline{\zeta}_{\gamma}$ and $\bar{\zeta}_{\gamma}$ such that the scaling factor $\gamma(t)$ satisfies the inequality

$$
\underline{\zeta}_{\gamma}<\gamma(t)<\bar{\zeta}_{\gamma}
$$

Proof: Since

$$
\gamma=\frac{\left\|q_{v}\right\|}{\left\|A q_{v}\right\|}
$$

the square of $\gamma(t)$ is

$$
\gamma^{2}=\frac{q_{v}^{T} q_{v}}{\left(A q_{v}\right)^{T} A q_{v}}=\frac{q_{v}^{T} q_{v}}{q_{v}^{T} A^{T} A q_{v}} .
$$

Since $A$ is of full rank, the symmetric matrix $A^{T} A$ is positive definite. Hence, the Rayleigh-Ritz theorem can be used to conclude that

$$
\lambda_{\min }\left(A^{T} A\right) q_{v}^{T} q_{v} \leq q_{v}^{T} A^{T} A q_{v} \leq \lambda_{\max }\left(A^{T} A\right) q_{v}^{T} q_{v}
$$


where $\lambda_{\min }\left(A^{T} A\right)$ and $\lambda_{\max }\left(A^{T} A\right)$ denote the minimal and maximal eigenvalues of $A^{T} A$, respectively. From (52) and (53), we can now conclude that

$$
\begin{aligned}
\frac{1}{\lambda_{\max }\left(A^{T} A\right)} & \leq \gamma^{2}=\frac{q_{v}^{T} q_{v}}{q_{v}^{T} A^{T} A q_{v}} \leq \frac{1}{\lambda_{\min }\left(A^{T} A\right)} \\
\sqrt{\frac{1}{\lambda_{\max }\left(A^{T} A\right)}} & \leq \gamma \leq \sqrt{\frac{1}{\lambda_{\min }\left(A^{T} A\right)}}
\end{aligned}
$$

\section{REFERENCES}

[1] K. Hosoda and M. Asada, "Versatile visual servoing without knowledge of true jacobian," in Proc. IEEE/RSJ Int. Conf. Intell. Robots Syst., 1994, pp. 186-193.

[2] M. Jagersand, O. Fuentes, and R. Nelson, "Experimental evaluation of uncalibrated visual servoing for precision manipulation," in Proc. Int. Conf. Robot. Automat., 1997, pp. 2874-2880.

[3] M. Shahamiri and M. Jagersand, "Uncalibrated visual servoing using a biased newton method for on-line singularity detection and avoidance," in Proc. IEEE/RSJ Int. Conf. Intell. Robots Syst., 2005, pp. 3953-3958.

[4] J. A. Piepmeier and H. Lipkin, "Uncalibrated eye-in-hand visual servoing," Int. J. Robot. Res., vol. 22, pp. 805-819, 2003.

[5] J. A. Piepmeier, G. V. McMurray, and H. Lipkin, "Uncalibrated dynamic visual servoing," IEEE Trans. Robot. Automat., vol. 24, no. 1, pp. 143-147, Jan. 2004.

[6] R. Kelly, "Robust asymptotically stable visual servoing of planar manipulator," IEEE Trans. Robot. Automat., vol. 12, no. 5, pp. 759-766, May 1996.

[7] B. Bishop and M. W. Spong, "Adaptive calibration and control of 2D monocular visual servo system," in Proc. IFAC Symp. Robot Control, 1997, pp. 525-530.

[8] L. Hsu and P. L. S. Aquino, "Adaptive visual tracking with uncertain manipulator dynamics and uncalibrated camera," in Proc. IEEE Conf. Decision Control, 1999, pp. 1248-1253.

[9] C. J. Taylor and J. P. Ostrowski, "Robust vision-based pose control," in Proc. IEEE Int. Conf. Robot. Automat., 2000, pp. 2734-2740.

[10] E. Zergeroglu, D. M. Dawson, M. de Queiroz, and A. Behal, "Visionbased nonlinear tracking controllers in the presence of parametric uncertainty," IEEE/ASME Trans. Mechatron., vol. 6, no. 3, pp. 322-337, 2001.

[11] W. E. Dixon, D. M. Dawson, E. Zergeroglu, and A. Behal, "Adaptive tracking control of a wheeled mobile robot via an uncalibrated camera system," IEEE Trans. Syst., Man, Cybern. B: Cybern., vol. 31, no. 3, pp. 341-352, Jun. 2001.

[12] A. Astolfi, L. Hsu, M. Netto, and R. Ortega, "Two solutions to the adaptive visual servoing problem," IEEE Trans. Robot. Automat., vol. 18, no. 3, pp. 387-392, Mar. 2002.

[13] A. Ruf, M. Tonko, R. Horaud, and H.-H. Nagel, "Visual tracking of an end-effector by adaptive kinematic prediction," in Proc. IEEE/RSJ Int. Conf. Intell. Robots Syst., 1997, pp. 893-898.

[14] Y. Liu, H. Wang, C. Wang, and K. Lam, "Uncalibrated visual servoing of robots using a depth-independent interaction matrix," IEEE Trans. Robotics, vol. 22, no. 4, pp. 804-817, Apr. 2006.

[15] G. Hu, W. MacKunis, N. Gans, and W. E. Dixon, "Adaptive homography-based visual servo control via an uncalibrated camera," in Proc. Amer. Control Conf., 2008, pp. 4791-4796.

[16] E. Malis and F. Chaumette, "Theoretical improvements in the stability analysis of a new class of model-free visual servoing methods," IEEE Trans. Robot. Automat., vol. 18, no. 2, pp. 176-186, Feb. 2002.

[17] Y. Fang, W. E. Dixon, D. M. Dawson, and J. Chen, "An exponential class of model-free visual servoing controllers in the presence of uncertain camera calibration," in Proc. IEEE Conf. Decision Control, 2003, pp. 5390-5395.
[18] G. Hu, N. Gans, and W. E. Dixon, "Quaternion-based visual servo control in the presence of camera calibration error," in Prof. IEEE MultiConf. Syst. Control, 2007, pp. 1492-1497.

[19] J. Chen, A. Behal, D. M. Dawson, and W. E. Dixon, "Adaptive visual servoing in the presence of intrinsic calibration uncertainty," in Proc. IEEE Conf. Decision Control, 2003, pp. 5396-5401.

[20] F. Chaumette, "Potential problems of stability and convergence in image-based and position-based visual servoing," in The Confluence of Vision and Control, ser. LNCIS Series, D. Kriegman, G. Hager, and A. Morse, Eds. Berlin, Germany: Springer-Verlag, 1998, vol. 237, pp. 66-78.

[21] P. Corke and S. Hutchinson, "A new partitioned approach to imagebased visual servo control," IEEE Trans. Robot. Automat., vol. 17, no. 4, pp. 507-515, Apr. 2001.

[22] B. Boufama and R. Mohr, "Epipole and fundamental matrix estimation using virtual parallax," in Proc. IEEE Int. Conf. Computer Vision, 1995, pp. 1030-1036.

[23] E. Malis and F. Chaumette, "2 1/2 D visual servoing with respect to unknown objects through a new estimation scheme of camera displacement," Int. J. Computer Vision, vol. 37, no. 1, pp. 79-97, 2000.

[24] O. Faugeras, Three-Dimensional Computer Vision: A Geometric Viewpoint. Cambridge, MA: MIT Press, 1993.

[25] R. Hartley and A. Zisserman, Multiple View Geometry in Computer Vision. New York: Cambridge University Press, 2000.

[26] A. Almansa, A. Desolneux, and S. Vamech, "Vanishing point detection without any a priori information," IEEE Trans. Pattern Anal. Machine Intell., vol. 25, no. 4, pp. 502-507, Apr. 2003.

[27] O. Faugeras and Q.-T. Luong, The Geometry of Multiple Images. Cambridge, MA: MIT Press, 2001.

[28] G. Hu, W. E. Dixon, S. Gupta, and N. Fitz-coy, "A quaternion formulation for homography-based visual servo control," in Proc. IEEE Int. Conf. Robot. Automat., 2006, pp. 2391-2396.

[29] M. Shuster, "A survey of attitude representations," J. Astronaut. Sci., vol. 41, no. 4, pp. 439-518, 1993.

[30] J. B. Kuipers, Quaternions and Rotation Sequences. Princeton, NJ: Princeton University Press, 1999.

[31] W. E. Dixon, A. Behal, D. M. Dawson, and S. Nagarkatti, Nonlinear Control of Engineering Systems: A Lyapunov-Based Approach. Boston, MA: Birkhäuser, 2003. 